\title{
Traumatisme majeur chez les personnes âgées : Les effets de l'âge sur l'évaluation des SU
}

\section{Par Elaine Cole}

$\mathrm{L}$ a population canadienne est vieillissante. En 2016, il y avait plus de personnes âgées de 65 ans et plus que de personnes âgées de 17 ans et moins (Statistique Canada, 2016). Ce changement de population est supposé continuer et, d'ici 2036, un quart des personnes au Canada seront âgées de 65 ans et plus (Association médicale canadienne, 2016). Les améliorations dans la santé, les aides sociales et le style de vie ont permis aux gens de vivre plus longtemps. Beaucoup d'entre eux restent relativement actifs ce qui, par conséquent, entraine une augmentation des lésions traumatiques pour ce groupe d'âge. La proportion de traumatisme chez les personnes âges arrivant aux services d'urgence (SU) augmente tous les ans (Kozar et al., 2015), et le nombre de personnes âgées avec d'importantes lésions devraient atteindre plus d'un tiers (39 $\%)$ des admissions générales en traumatologie d'ici 2050 (Banks \& Lewis, 2013).

Contrairement aux soins de traumatologie pédiatrique, la gestion optimale des traumatismes chez les personnes âgées manque présentement de lignes directrices en termes de traitements axés sur lâge et de protocoles cliniques. De nombreux patients âgées sérieusement blessés réagissent différemment face aux chocs et aux lésions, ce qui entraîne des défis uniques pour l'équipe de traumatologie des SU (Bradburn et al., 2012). Le vieillissement est souvent associé à un déclin progressif des fonctions physiologiques ce qui, à terme, modifie les capacités du corps à répondre efficacement face aux lésions traumatiques (Panda et al., 2009), surtout chez les patients âgés fragiles souffrant de plusieurs problèmes de santé (Joseph et al., 2017). Les patients âgés en traumatologie sont régulièrement mal triés (Ichwan et al., 2015; Reske-Nielsen \& Medzon, 2016) et des maladies co-existantes ou des médicaments peuvent alterer les signes ou les symptômes des lésions (Llompart-Pou, Perez-Barcena, Chico-Fernandez, Sanchez-Casado, \& Raurich, 2017). Ces facteurs peuvent entraîner des retards dans l'accès à des soins spécialisés ou à de mauvais diagnostics, dont tous deux contribuent à de mauvais résultats après des lésions traumatiques (Hranjec, Sawyer, Young, Swenson, \& Calland, 2012). Même si l'intégration des services de traumatologie gériatrique au Canada (Wong et al., 2017) et aux É.U. (Wiles, Day, \& Harris, 2016) sont connus pour améliorer les résultats, cela demeure une aspiration pour de nombreux hôpitaux et la responsabilité de la gestion des personnes âgées souffrant d'un traumatisme incombe à l'équipe des SU.

Ce document discutera de certaines différences liées à lâge pouvant influencer l'évaluation et la gestion de patients âgés souffrant de traumatisme dans les SU. Aux fins du présent document, les mots « personnes âgées 》 ou «âgé » décrivent des patients âgés de 65 ans et plus, tout en reconnaissant que cela peut être différent selon les environnements cliniques.

\section{Mécanismes des lésions chez les personnes âgées souffrant de traumatisme}

Comparativement aux adultes plus jeunes, les personnes âgées peuvent subir d'importantes lésions à partir de mécanismes relativement mineurs (Cryer, 2013). Le traumatisme contondant est la cause dominante chez ce groupe d'âge (Adams et al., 2012; Dinh, Roncal, Byrne, \& Petchell, 2013), et un quart des traumatismes sévères proviennent d'accidents de voiture (Bonne \& Schuerer, 2013). Cependant, il est devenu de plus en plus évident que la plupart des mécanismes courants de lésions chez les personnes âgées sont en rapport avec des chutes (Gillespie et al., 2012; Kozar et al., 2015; TARN, 2017).

De nombreuses « personnes âgées plus jeunes » et actives tendent à tomber à l'extérieur (Kelsey, Procter-Gray, Hannan, \& Li, 2012; Kim, 2016), mais de nombreuses personnes plus âgées tombent à l'intérieur à cause de chutes à niveau bas niveau ou à faible énergie (Gelbard et al., 2014; TARN, 2017). Même si les chutes à bas niveau sont généralement moins importantes pour les patients plus jeunes, elles sont cependant la principale cause en termes de lésions cérébrales traumatiques (LCT) et de mortalité chez les personnes plus âgées. (Carpenter et al., 2014; Llompart-Pou et al., 2017). Malgré cela, de nombreux triages ou protocoles en traumatologie dans les SU n'ont présentement pas les chutes à bas niveau comme critère d'activation. L'utilisation de Warfarine (Coumadin) est courante chez les personnes âgées et le besoin d'inversion d'urgence pour minimiser l'hémorragie cérébrale (et d'autres) est bien documenté. Cependant, les anticoagulants oraux directs (ACOD) sont prescrits à de nombreux patients (Wood et al., 2017) et contrairement au Wafarine, pas tous les ACOD - comme le Rivaroxaban - ont des agents réversifs (Barletta et al., 2017; Kobayashi et al., 2017). Les risques de résultats négatifs ou de mortalité après une chute augmentent chez les patients âgés anticoagulés et souffrant d'un traumatisme (Boltz, Podany, Hollenbeak, \& Armen, 2015). Pour un(e) infirmier/infirmière des $\mathrm{SU}$, les chutes chez les patients âgés indiquent la probabilité d'une lésion sévère, surtout avec la présence d'anticoagulants oraux. La cause sous-jacente d'une chute doit être également prise en compte.

Évaluation initiale du traumatisme chez les personnes âgées et les effets du vieillissement

La physiologie du processus de vieillissement signifie que la réponse physiologique «normale » en réponse à une lésion peut ne pas être la même chez des patients plus âgés. Les maladies de comorbidité peuvent compliquer les situations, et environ $75 \%$ des personnes âgées au Canada ont signalé avoir eu une ou plusieurs conditions chroniques (Institut canadien d'information sur la santé, 2014). Les maladies de comorbidité (ou co-existantes) et les médicaments connexes compliquent l'évaluation « normale » d'un traumatisme, où les bêta-bloquants 
ou les simulateurs cardiaques peuvent cacher des signes vitaux anormaux comme la tachycardie (Bonne \& Schuerer, 2013; Stevens \& Torke, 2016).

\section{Évaluation des voies respiratoires et immobilisation de la colonne cervicale}

Une évaluation et gestion opportunes des voies respiratoires sont essentielles pour tout patient souffrant d'un traumatisme. Cependant, en raison de corps étrangers, comme des prothèses dentaires ou perte de dents, il existe un risque accru d'une obstruction possible des voies respiratoires chez les personnes âgées (Dalton, Rushing, Escott \& Monroe 2015). En outre, les exigences relatives à une immobilisation appropriée de la colonne cervicale peuvent s'avérer être difficiles en raison de nombreux facteurs, comme la cyphose, la lordose ou les maladies dégénératives du cou et aucune tentative à obliger le patient à se mettre en position couchée ne devrait être faite (Rao, Phan, Mobbs, Wilson \& Ball 2016). Pour terminer, il y a une augmentation de la fréquence des lésions cachées (ou non détectées) de la colonne cervicale chez les personnes âgées blessées à la tête (Kozar et al., 2015). Par conséquent, si un traumatisme crânien est probable, la colonne cervicale devrait être incluse lors d'une tomodensitométrie crânienne.

\section{Évaluation respiratoire}

Le vieillissement est associé à une baisse de la réserve respiratoire, principalement parce que le tissu pulmonaire devient de plus en plus inélastique et les fonctions alvéolaires diminuent (Johnson, Botros, Groban, \& Bryan, 2015). Les maladies pulmonaires chroniques, comme l'emphysème ou la MPOC, augmentent les risques d'insuffisance respiratoire chez les personnes âgées. En outre, comme la cage thoracique devient plus rigide, même les blessures les plus « mineures » à la poitrine, ou des fractures à une côte, peuvent entraîner d'importantes complications respiratoires (Wardhan, 2013). Les changements physiologiques du vieillissement, associés au système respiratoire et aux maladies de comorbidité, peuvent entraîner une baisse des niveaux de saturation en oxygène ( $<95 \%$ sur l'air) dans un état pré-lésionnel. Cependant, de faibles saturations en $\mathrm{Sa} 02$ chez les personnes âgées souffrant d'un traumatisme doivent être considérées comme anormales jusquà ce que la lésion soit écartée. L'exposition aux radiations n'est pas une préoccupation chez ce groupe d'âge et une radio du thorax (ou une tomodensitométrie) est indispensable pour détecter des lésions au thorax.

\section{Évaluation cardiovasculaire}

Un traumatisme chez les personnes âgées est considéré de façon anecdotique comme des « lésions crâniennes ou des fractures de la hanche $\gg$. Cependant, une récente étude Britannique a indiqué qu'une importante hémorragie est un lourd fardeau chez les patients plus âgé souffrant d'un traumatisme (Stanworth et al., 2016). Durant une évaluation de traumatisme cardiovasculaire dans les SU, des signes vitaux anormaux (comme la tachycardie et l'hypertension) sont généralement des indicateurs pour l'équipe de traumatologie (Heffernan et al., 2010). Cependant, la TA systolique (TAS) et le rythme cardiaque ne sont pas d'une grande utilité pour prédire de sérieuses lésions chez les personnes âgées (Newgard et al., 2014). Plus de $50 \%$ de la population gériatrique souffrent d'hypertension et de maladies cardiovasculaires sous-jacentes (Bonne \& Schuerer, 2013) où un TAS « normale » peut être de $150 \mathrm{mmHg}$ ou plus élevé. Il est, par conséquent, difficile d'évaluer l'hypertension chez ces patients, et des signes vitaux généralement acceptés, comme une TAS $<90 \mathrm{mmHg}$, peuvent être extrêmement faibles ou en phase préterminale pour certaines personnes plus âgées (Heffernan et al., 2010). Les résultats des adultes âgés entre 65 et 95 ans varieront grandement avec le vieillissement puisqu'il n'y a pas de véritable seuil unique en termes d'hypertension pour les personnes âgées.

Cependant, de récents rapports suggèrent que des valeurs plus élevées de TAS $<110$ to $117 \mathrm{~mm} \mathrm{Hg}$ sont plus sensibles pour prédire l'hypertension, des lésions sévères et la mortalité dans un traumatisme plus ancien (Brown et al., 2015; Newgard et al., 2014; Oyetunji et al., 2011). Pour aider avec l'évaluation d'hémorragie chez les personnes âgées, les marqueurs d'hypoperfusion de laboratoire, comme le lactate ou le déficit de base, peuvent être utiles. Un lactate veineux élevé $\geq 2.5$ $\mathrm{mmol} / \mathrm{L}$ permet de mieux identifier l'instabilité hémodynamique que la TAS dans un traumatisme plus ancien (Salottolo, Mains, Offner, Bourg, \& Bar-Or, 2013), et le lactate élevé ( $\geq$ $2.0 \mathrm{mmol} / \mathrm{L}$ ) dans un traumatisme chez les personnes âgées est supposé être un facteur à risque pour de mauvais résultats (Vanzant et al., 2015). L'American College of Surgeons suggère qu'un déficit de base devrait être accéléré dans l'évaluation de traumatisme gériatrique (Cryer, 2013). Les valeurs d'un déficit de base de $\geq 6 \mathrm{mEq} / \mathrm{L}$ (Base Excess $\leq-6 \mathrm{mEq} / \mathrm{L}$ ) sont des marqueurs de lésions sévères et $60 \%$ de la mortalité chez les personnes âgées souffrant d'un traumatisme (Calland et al., 2012; Davis \& Kaups, 1998).

\section{Évaluation neurologique}

L'évaluation neurologique précise chez les personnes âgées victimes d'un traumatisme peut s'avérer être assez complexe avec des déficiences cognitives préexistantes, comme la démence ou Alzheimer, ou des facteurs en rapport avec l'âge comme la perte auditive. L'échelle de Glasgow est utilisée pour déterminer l'étendue des lésions neurologiques chez les patients souffrant d'un traumatisme cérébral et d'identifier ceux nécessitant des soins neurochirurgicaux. Cependant, cette évaluation peut avoir un usage limité chez les personnes âgées (Salottolo, Levy, Slone, Mains, \& Bar-Or, 2014). Des études suggèrent que l'Échelle de Glasgow dans un traumatisme plus ancien peut ne pas refléter la sévérité d'un traumatisme cérébral, et les résultats se sont retrouvés être plus élevés chez les personnes âgées que chez des patients plus jeunes mais avec des lésions de même gravité (Kehoe, Rennie, \& Smith, 2015; Kehoe et al., 2016). Ceci est peut-être dû à l'atrophie cérébrale, créant plus d'espace à l'intérieur du crâne pour permettre à un hématome de croître, ou le fait que les hématomes sous-duraux - courants chez les personnes âgées - évoluent plus lentement et réduisent l'Échelle de Glasgow à une étape plus tardive (Kehoe et al., 2016; Salottolo et al., 2014). Une tomodensitométrie cérébrale précoce est essentielle pour réussir à identifier et à catégoriser le traumatisme cérébral (Carney, 2016). Cependant, un âge plus avancé est souvent associé avec une tomodensitométrie plus tardive (Kirkman et al., 2013; TARN, 2017), ce qui peut entraîner, en partie, une incapacité à reconnaître une baisse de l'Échelle de Glasgow. Une fois de plus, des variations dans l'âge compliquent la possibilité de donner une 
valeur précise pouvant indiquer un traumatisme cérébral, mais un score de Glasgow de 14 ou moins peut être prédicatif d'une lésion sérieuse (Newgard et al., 2014) chez les personnes âgées, jusqu’à preuve du contraire.

En résumé, l'identification d'une lésion sérieuse chez les personnes âgées victimes d'un traumatisme est difficile. Les changements physiologiques associés avec l'âge et les maladies de comorbidité compliquent l'évaluation du traumatisme des personnes âgées. Avec l'augmentation du nombre de personnes âgées, il devient essentiel pour les infirmiers/infirmières des SU d'évaluer adéquatement ce groupe d'âge.

Les messages clés pour l'évaluation de traumatisme chez les personnes âgées dans les SU sont :

- Quelle que soit l'apparence « jeune » d'un patient âgé victime d'un traumatisme, il y aura des changements physiologiques sous-jacents pouvant masquer les signes « normaux » de lésions.

- Des chutes à bas niveau peuvent entraîner des lésions sérieuses chez les personnes âgées, et un examen approfondi est obligatoire.

- Les anticoagulants oraux peuvent compliquer les lésions et aggraver les résultats. Des diagnostics et des conseils précoces sur un renversement sont essentiels.

- Les signes vitaux peuvent ne pas correspondre à des paramètres $\ll$ normaux $»$ :
- De faibles saturations en oxygène devraient être considérées comme une hypoxie en rapport avec un traumatisme, jusquà preuve du contraire. Vérifiez les gaz sanguins artériels!

- Une TAS de $<120 \mathrm{mmHg}$ peut s'avérer être de l'hypertension chez les personnes âgées.

- Un lactate de $\geq 2.0 \mathrm{mmol} / \mathrm{L}$ ou un déficit de base de $\geq 6 \mathrm{mEq} / \mathrm{L}$ peuvent être de meilleurs marqueurs d'une lésion sévère qu'un TAS.

- Un score de Glasgow «normale » (14-15) dans une lésion cérébrale d'une personne âgée n'exclut pas un traumatisme cérébral.

\section{Au sujet de l'auteure}

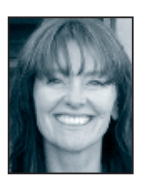

Dr Elaine Cole, PhD, MSc, Diplôme écoles supérieures (éducation), BSc, RN. Mes expériences professionnelles se situent les domaines des $S U$ et des soins infirmiers en soins intensifs. Après avoir travaillé plusieurs années en tant que spécialiste dans les SU, j'ai terminé mon doctorat en novembre 2014 (examinant le lien entre l'hémorragie et l'infection après une sérieuse lésion). Présentement, je travaille en tant que Directrice de recherche et d'innovation pour le London Major Trauma System (www.londontraumasystem.org), où je soutiens l'innovation clinique et la recherche collaborative dans la Région du grand Londres. Je suis également une des conseillères et dirigeantes de module pour le MSc Trauma Sciences à l'Université Queen Mary de Londres.

\section{RÉFÉRENCES}

Adams, S.D., Cotton, B.A., McGuire, M.F., Dipasupil, E., Podbielski, J.M., Zaharia, A., ... Holcomb, J.B. (2012). Unique pattern of complications in elderly trauma patients at a Level I trauma center. J Trauma Acute Care Surg, 72(1), 112-118. doi:10.1097/ TA.0b013e $318241 \mathrm{f} 073$

Banks, S.E., \& Lewis, M.C. (2013). Trauma in the elderly: Considerations for anesthetic management. Anesthesiol Clin, 31(1), 127-139. doi:10.1016/j.anclin.2012.11.004

Barletta, J.F., Hall, S., Sucher, J.F., Dzandu, J.K., Haley, M., \& Mangram, A.J. (2017). The impact of pre-injury direct oral anticoagulants compared to warfarin in geriatric G-60 trauma patients. European Journal of Trauma and Emergency Surgery, 43(4), 445-449. doi:10.1007/s00068-017-0772-z

Boltz, M.M., Podany, A.B., Hollenbeak, C.S., \& Armen, S.B. (2015). Injuries and outcomes associated with traumatic falls in the elderly population on oral anticoagulant therapy. Injury, 46(9), 1765-1771. doi:10.1016/j.injury.2015.06.013

Bonne, S., \& Schuerer, D.J. (2013). Trauma in the older adult: epidemiology and evolving geriatric trauma principles. Clin Geriatr Med, 29(1), 137-150. doi:10.1016/j.cger.2012.10.008

Bradburn, E., Rogers, F.B., Krasne, M., Rogers, A., Horst, M.A., Beelen, M.J., \& Miller, J.A. (2012). High-risk geriatric protocol: Improving mortality in the elderly. J Trauma Acute Care Surg, 73(2), 435-440. doi:10.1097/TA.0b013e31825c7cf4

Brown, J.B., Gestring, M.L., Forsythe, R.M., Stassen, N.A., Billiar, T.R., Peitzman, A.B., \& Sperry, J.L. (2015). Systolic blood pressure criteria in the National Trauma Triage Protocol for geriatric trauma: 110 is the new 90. J Trauma Acute Care Surg, 78(2), 352359. doi: $10.1097 /$ ta.0000000000000523

Calland, J.F., Ingraham, A.M., Martin, N., Marshall, G.T., Schulman, C.I., Stapleton, T., \& Barraco, R.D. (2012). Evaluation and management of geriatric trauma: An Eastern Association for the
Surgery of Trauma practice management guideline. J Trauma Acute Care Surg, 73(5, Suppl. 4), S345-350. doi:10.1097/ TA.0b013e318270191f

Carney, N., Totten, A., O'Reilly, C., et al. (2016). Brain Trauma Foundation. Guidelines for the management of severe traumatic brain injury (4th edition). https://braintrauma.org/ uploads/03/12/Guidelines_for_Management_of_Severe TBI_4th_Edition.pdf

Carpenter, C.R., Avidan, M.S., Wildes, T., Stark, S., Fowler, S.A., \& Lo, A.X. (2014). Predicting geriatric falls following an episode of emergency department care: A systematic review. Acad Emerg Med, 21(10), 1069-1082. doi:10.1111/acem.12488

Canadian Medical Association. (2016). The state of seniors' healthcare in Canada. https://www.cma.ca/En/Lists/Medias/the-state-ofseniors-health-care-in-canada-september-2016.pdf

Canadian Institute for Health Information. (2014). Health care in Canada, 2011. A focus on seniors and aging. Ottawa: Author. https://secure.cihi.ca/free_products/ HCIC_2011_seniors_report_en.pdf

Cryer, H.G., Calland, J.F., Chow, W., Davis, M., Hemmila, M., Kosar, R., Lopez, S., ... Wong, C. (2013). American College of Surgeons Trauma Quality Improvement Geriatric Trauma Management Guidelines. https://www.facs.org/ /media/files/quality\%20 programs/trauma/tqip/geriatric\%20guide\%20tqip.ashx

Dalton, T., Rushing, M.R, Escott, M.E., \& Monroe, B.J. (2015) Complexities of geriatric trauma patients. JEMS. http://www.jems. com/articles/print/volume-40/issue-11/features/complexitiesof-geriatric-trauma-patients.html

Davis, J.W., \& Kaups, K.L. (1998). Base deficit in the elderly: A marker of severe injury and death. J Trauma, 45(5), 873-877.

Dinh, M.M., Roncal, S., Byrne, C.M., \& Petchell, J. (2013). Growing trend in older patients with severe injuries: Mortality and 
mechanisms of injury between 1991 and 2010 at an inner city major trauma centre. ANZ J Surg, 83(1-2), 65-69. doi:10.1111/j.1445-2197.2012.06180.x

Gelbard, R., Inaba, K., Okoye, O.T., Morrell, M., Saadi, Z., Lam, L., ... Demetriades, D. (2014). Falls in the elderly: A modern look at an old problem. Am J Surg, 208(2), 249-253. doi:10.1016/j. amjsurg.2013.12.034

Gillespie, L.D., Robertson, M.C., Gillespie, W.J., Sherrington, C., Gates, S., Clemson, L.M., \& Lamb, S.E. (2012). Interventions for preventing falls in older people living in the community. Cochrane Database Syst Rev(9), Cd007146. doi:10.1002/14651858. CD007146.pub3

Heffernan, D.S., Thakkar, R.K., Monaghan, S.F., Ravindran, R., Adams, C.A., Jr., Kozloff, M.S., ... Cioffi, W.G. (2010). Normal presenting vital signs are unreliable in geriatric blunt trauma victims. $J$ Trauma, 69(4), 813-820. doi:10.1097/TA.0b013e3181f41af8

Hranjec, T., Sawyer, R.G., Young, J.S., Swenson, B.R., \& Calland, J.F. (2012). Mortality factors in geriatric blunt trauma patients: Creation of a highly predictive statistical model for mortality using 50,765 consecutive elderly trauma admissions from the National Sample Project. Am Surg, 78(12), 1369-1375.

Ichwan, B., Darbha, S., Shah, M.N., Thompson, L., Evans, D.C., Boulger, C.T., \& Caterino, J.M. (2015). Geriatric-specific triage criteria are more sensitive than standard adult criteria in identifying need for trauma center care in injured older adults. Ann Emerg Med, 65(1), 92-100.e103. doi:10.1016/j.annemergmed.2014.04.019

Johnson, K.N., Botros, D.B., Groban, L., \& Bryan, Y.F. (2015). Anatomic and physiopathologic changes affecting the airway of the elderly patient: Implications for geriatric-focused airway management. Clin Interv Aging, 10, 1925-1934. doi:10.2147/cia. s93796

Joseph, B., Orouji Jokar, T., Hassan, A., Azim, A., Mohler, M.J., Kulvatunyou, N., ... Rhee, P. (2017). Redefining the association between old age and poor outcomes after trauma: The impact of frailty syndrome. J Trauma Acute Care Surg, 82(3), 575-581. doi:10.1097/ta.0000000000001329

Kehoe, A., Rennie, S., \& Smith, J.E. (2015). Glasgow Coma Scale is unreliable for the prediction of severe head injury in elderly trauma patients. Emerg Med J, 32(8), 613-615. doi:10.1136/ emermed-2013-203488

Kehoe, A., Smith, J.E., Bouamra, O., Edwards, A., Yates, D., \& Lecky, F. (2016). Older patients with traumatic brain injury present with a higher GCS score than younger patients for a given severity of injury. Emerg Med J, 33(6), 381-385. doi:10.1136/ emermed-2015-205180

Kelsey, J.L., Procter-Gray, E., Hannan, M.T., \& Li, W. (2012). Heterogeneity of falls among older adults: Implications for public health prevention. Am J Public Health, 102(11), 2149-2156. doi:10.2105/ajph.2012.300677

Kim, S.H. (2016). Risk factors for severe injury following indoor and outdoor falls in geriatric patients. Arch Gerontol Geriatr, 62, 75-82. doi:10.1016/j.archger.2015.10.003

Kirkman, M.A., Jenks, T., Bouamra, O., Edwards, A., Yates, D., \& Wilson, M.H. (2013). Increased mortality associated with cerebral contusions following trauma in the elderly: Bad patients or bad management? J Neurotrauma, 30(16), 1385-1390. doi:10.1089/ neu.2013.2881

Kobayashi, L., Barmparas, G., Bosarge, P., Brown, C.V., Bukur, M., Carrick, M.M., ... Coimbra, R. (2017). Novel oral anticoagulants and trauma: The results of a prospective American Association for the Surgery of Trauma Multi-Institutional Trial. J Trauma Acute Care Surg, 82(5), 827-835. doi:10.1097/ta.0000000000001414

Kozar, R.A., Arbabi, S., Stein, D.M., Shackford, S.R., Barraco, R.D., Biffl, W.L., ... Luchette, F. (2015). Injury in the aged: Geriatric trauma care at the crossroads. J Trauma Acute Care Surg, 78(6), 1197-1209. doi:10.1097/ta.0000000000000656
Llompart-Pou, J.A., Perez-Barcena, J., Chico-Fernandez, M., SanchezCasado, M., \& Raurich, J.M. (2017). Severe trauma in the geriatric population. World J Crit Care Med, 6(2), 99-106. doi:10.5492/ wjccm.v6.i2.99

Newgard, C.D., Richardson, D., Holmes, J.F., Rea, T.D., Hsia, R.Y., Mann, N.C., ... Haukoos, J.S. (2014). Physiologic field triage criteria for identifying seriously injured older adults. Prehosp Emerg Care, 18(4), 461-470. doi:10.3109/10903127.2014.9127 07

Oyetunji, T.A., Chang, D.C., Crompton, J.G., Greene, W.R., Efron, D.T., Haut, E.R., ... Haider, A.H. (2011). Redefining hypotension in the elderly: Normotension is not reassuring. Arch Surg, 146(7), 865-869. doi:10.1001/archsurg.2011.154

Panda, A., Arjona, A., Sapey, E., Bai, F., Fikrig, E., Montgomery, R.R., ... Shaw, A.C. (2009). Human innate immunosenescence: Causes and consequences for immunity in old age. Trends Immunol, 30(7), 325-333. doi:10.1016/j.it.2009.05.004

Rao, P.J., Phan, K., Mobbs, R.J, Wilson, D., \& Ball, B. (2016). Cervical spine immobilization in the elderly population. J Spine Surg., 2(1), 41-46.

Reske-Nielsen, C., \& Medzon, R. (2016). Geriatric Trauma. Emerg Med Clin North Am, 34(3), 483-500. doi:10.1016/j.emc.2016.04.004

Salottolo, K., Levy, A.S., Slone, D.S., Mains, C.W., \& Bar-Or, D. (2014). The effect of age on Glasgow Coma Scale score in patients with traumatic brain injury. JAMA Surg, 149(7), 727-734. doi:10.1001/jamasurg.2014.13

Salottolo, K.M., Mains, C.W., Offner, P.J., Bourg, P.W., \& Bar-Or, D. (2013). A retrospective analysis of geriatric trauma patients: Venous lactate is a better predictor of mortality than traditional vital signs. Scand J Trauma Resusc Emerg Med, 21, 7. doi:10.1186/1757-7241-21-7

Stanworth, S.J., Davenport, R., Curry, N., Seeney, F., Eaglestone, S., Edwards, A., ... Brohi, K. (2016). Mortality from trauma haemorrhage and opportunities for improvement in transfusion practice. Br J Surg. doi:10.1002/bjs. 10052

Statistics Canada. (2016). Annual demographic estimates: Canada, Provinces and Territories (91-215-X). http://www5.statcan.gc.ca/olc-cel/olc. action?ObjId=91-215-X\&ObjType $=2 \&$ lang $=$ en $\&$ limit $=0$

Stevens, C.L., \& Torke, A.M. (2016). Geriatric trauma: A clinical and ethical review. J Trauma Nurs, 23(1), 36-41. doi:10.1097/ jtn.0000000000000179

Trauma Audit and Research Network [TARN]. (2017). Major trauma in older people. https://www.tarn.ac.uk/content/downloads/3793/ Major\%20Trauma\%20in\%20Older\%20People\%202017.pdf

Vanzant, E.L., Hilton, R.E., Lopez, C.M., Zhang, J., Ungaro, R.F., Gentile, L.F., ... Efron, P.A. (2015). Advanced age is associated with worsened outcomes and a unique genomic response in severely injured patients with hemorrhagic shock. Crit Care, 19, 77. doi:10.1186/s13054-015-0788-x

Wardhan, R. (2013). Assessment and management of rib fracture pain in geriatric population: An ode to old age. Curr Opin Anaesthesiol, 26(5), 626-631. doi:10.1097/01.aco.0000432516.93715.a7

Wiles, L.L., Day, M.D., \& Harris, L. (2016). Delta alerts: Changing outcomes in geriatric trauma. J Trauma Nurs, 23(4), 189-193. doi: $10.1097 /$ jtn.0000000000000215

Wong, C.L., Al Atia, R., McFarlan, A., Lee, H.Y., Valiaveettil, C., \& Haas, B. (2017). Sustainability of a proactive geriatric trauma consultation service. Can J Surg, 60(1), 14-18.

Wood, B., Nascimento, B., Rizoli, S., Sholzberg, M., McFarlan, A., Phillips, A., \& Ackery, A.D. (2017). The anticoagulated trauma patient in the age of the direct oral anticoagulants: A Canadian perspective. Scand J Trauma Resusc Emerg Med, 25(1), 76. doi:10.1186/s13049-017-0420-y 